\title{
Persistence of Pleural Effusions and Empyemas after Pneumococcal Conjugate Vaccine Implementation in Uruguay
}

\author{
Miguel Estevan ${ }^{1}$, Luis Martínez ${ }^{2}$, Edith Arreisengor ${ }^{3}$, Maria Hortal $^{4}$ \\ ${ }^{1}$ Radiology Departments, Children’s Hospital, Montevideo, Uruguay; ${ }^{2}$ Department of Pediatric, Paysandú Hospital, Paysandú, Uru- \\ guay; ${ }^{3}$ Pediatric Service, Salto Medical Society, Salto, Uruguay; ${ }^{4}$ Basic Sciences Program, National University, Montevideo, Uru- \\ guay. \\ Email: *marujahortal@gmail.com
}

Received September $22^{\text {nd }}$, 2012; revised October 23 $3^{\text {rd }}, 2012$; accepted November $3^{\text {rd }}$, 2012

\begin{abstract}
In Uruguay a post pneumococcal conjugate vaccine implementation surveillance of hospitalized children with pneumonia showed an increase of complicated pneumonias, while uncomplicated pneumonias decreased. Out of 151 pleural effusions, 62 were empyemas requiring drainage, the rest of cases were treated with antibiotics with a favorable outcome. Patient's vaccinated status varied. Pneumococcal etiology was poorly documented. The few identified serotypes were 1 and 3, a fact that urges PCV13 use for their control.
\end{abstract}

Keywords: Pneumonia; Pleural Effusion; Pneumococcal Infections; Conjugate Vaccine

\section{Introduction}

Pneumonia with pleural effusion or empyema complications were seldom observed in developed countries. However, an increasing tendency of these complications was seen in hospitalized children with pneumonia, both before and after the implementation of pneumococcal conjugate vaccine (PCV), just like several publications from different countries describe [1,2]. In Uruguay, 20012004 data from patients aged 0 to 59 months were diverse. Patients with pneumonia complicated with parapneumonic effusion referred for treatment at the Children's Pediatric Hospital represented $47 \%$ of the hospitalized pneumococcal pneumonias [3,4], whereas a population-based study of 831 patients hospitalized with consolidated pneumonias, conducted during the same period of time, reported 102 pleural effusions (57 empyemas), representing a relative frequency of $12.3 \%$ [5].

A 2005/2008 information gap hampered the possibility of tracing the tendency of complicated pneumonias before PCV7 introduction. Nevertheless, a database from the Pediatric Service of the Paysandú Public Hospital, provided 2005/2008 information on consolidated pneumonia admissions, enabling the assessment of a discrete increase of pleural effusions/empyemas among inpatients of that institution: (L: Martinez, personal communica-

*Corresponding author. tion). In Uruguay, a population-based surveillance on consolidated pneumonias occurrence in hospitalized children 0 to 14 years of age provided data from January 2009 to December 2011. Thus, it was possible to assess the epidemiological behavior of complicated pneumonias after PCV7 and PCV13 implementation, and to describe its clinical impact and outcome.

The aim of this report is to analyze the temporal evolution of pneumonias and its complications, characterized by a relative increase of parapneumonic effusions, while pneumococcal conjugated vaccine program was in progress.

\section{Methods}

The present study applied the same methodology employed in the pre-vaccination population-based surveillance baseline [5]. All hospitalized patients who had a chest X-ray to confirm pneumonia were eligible. X-rays were digitalized and interpreted by a reference pediatric radiologist according WHO criteria [6]. Ultrasound documented pleural effusions. Clinical/radiographic variables were recorded for patients aged 0 to 14 years. Vaccination certificate provided data on vaccination status of inpatients who had received PCV at 2, 4 and 12 months of age. Bacterial etiology was investigated in blood and/or in pleural fluid. This study was approved by the National Ethics Committee from the Ministry of Health. 


\section{Results}

Parapneumonic effusion and empyema were the most frequent bacterial pneumonia complications among the hospitalized children during both surveillance periods (2001/04 and 2009/11). Table 1 shows the number of consolidated pneumonias and pleural effusions pre and post PCV implementation in patients under 5 years of age and in those aged 0 through 14 years. A progressive decrease of the rate for consolidated pneumonia was observed in the post vaccination period. Despite this reduction, a relative high proportion of pleural effusions persisted.

Sixty two out of the 151 pleural effusions (41\%) were empyemas that required drainage, while the rest of them had limited clinical relevance and were successfully managed with antibiotics. No decortication was needed for the empyema cases and only three patients required intensive care assistance. Average stay in hospital was 15 days for the empyemas and 6.1 days for the uncomplicated pleural effusions. No seasonal variation was associated with the occurrence of complicated pneumonias as cases were detected every month of the surveyed years.

The vaccination status of patients with complicated pneumonia varied. Out of 47 patients 0 to 14 years who were enrolled during 2011, 25 were non vaccinated (17 had no vaccine indication; 8 were unvaccinated). As to the remaining 22 patients, 9 of them were fully vaccinated with PCV7, 8 had received PCV13 primary doses and 2 the booster dose.

The etiology of the 2011 complicated pneumonias was poorly documented. S. pneumoniae etiology was recognized by antigen detection in the fluid of 3 empyemas, and by isolation from blood or pleural fluid of 5 patients. Also, a pleural fluid culture yielded one $H$. influenzae b. Only two of the isolates were available for typing, where serotypes 1 and 3 were identified.

\section{Discussion}

The ongoing surveillance of hospitalized pneumonias in children enabled us to document a relative increase of pneumonias complicated with pleural effusion, while uncomplicated pneumonias decreased. This phenomena have been observed in different settings, even before PCV introduction [7]. Its clinical impact, characterized by a favorable outcome, prompted the introduction of PCV13 with its extended formulation, probably able to control the dynamic relation between disease incidence and vaccine coverage [8].

The introduction of PCVs was associated with epidemiological changes in serotype prevalence. Serotype 14, the most important cause of pneumonias in children under 24 months of age, was efficiently controlled. PCV7 probably reduced pneumonia incidence mostly associated with serotypes causing uncomplicated pneumonias, although it favored the persistence of other serotypes not included in its formulation [9]. Serotypes 5 and 1 respectively the second and third ones in frequency among Uruguayan children, had a different epidemiological behavior. Both serotypes were seldom identified because their susceptibility to antibiotics interfered with the cultures, and were more unpredictable because they caused small outbreaks or cluster of cases.

Serotype 1 was frequently recognized to be associated with pleural effusions in different countries. Although PCV13 was expected to control this and the other five additional serotypes that are included in its formulation, time is required for having the 2010 cohort fully vaccinated. Also, higher coverage is needed to reduce the carriage of the recently added six serotypes. The majority of the complicated pneumonias occurred in non vaccinated patients, patients vaccinated with PCV7, or patients who were too old to be vaccinated.

It is crucial to detect the agents involved in remaining complicated pneumonias, as other bacterial infectious like Staphylococcus aureus might contribute to persis-

Table 1. Consolidated pneumonias and pleural effusion: frequency and incidence by study periods (2001/04 and 2009/11).

\begin{tabular}{|c|c|c|c|c|c|c|}
\hline Period & Age & Consolidated pneumonia & Pleural effusion & \% Pleural effusion & $\begin{array}{l}\text { Pl. effusion } \\
\text { incidence }\end{array}$ & $\begin{array}{c}\text { Pneumonia } \\
\text { incidence }\end{array}$ \\
\hline \multirow[t]{2}{*}{$2001 / 04$} & $<5$ years & 831 & 102 & 12.3 & 165 & 1341 \\
\hline & 0 - 14 years & 1060 & 133 & 12.5 & 66 & 527 \\
\hline \multirow[t]{2}{*}{2009} & $<5$ years & 232 & 43 & 18.5 & 208 & 1123 \\
\hline & 0 - 14 years & 330 & 67 & 20.3 & 100 & 492 \\
\hline \multirow[t]{2}{*}{2010} & $<5$ years & 166 & 30 & 18.1 & 146 & 804 \\
\hline & 0 - 14 years & 224 & 47 & 20.9 & 70 & 334 \\
\hline \multirow[t]{2}{*}{2011} & $<5$ years & 148 & 27 & 18.2 & 131 & 717 \\
\hline & 0 - 14 years & 236 & 47 & 19.9 & 70 & 352 \\
\hline
\end{tabular}


tence [10]. It is also relevant to serotype the invasivepneumococci in order to confirm the serotypes controlled by PCV13, or to recognize replacements by non-vaccine serotypes. This remaining burden is difficult to estimate and requires the support of microbiological diagnostic methods with greater accuracy.

\section{Acknowledgements}

Our recognition to Dr A. Cané for his permanent academic and technical contribution, and to Prof O. Bello for promoting local research. To Nurses G. Barrios, M. Alvez and to all the health personnel from the surveyed institutions.

\section{REFERENCES}

[1] S. T. Li and D. J. Tancredi, "Empyema Hospitalization Increased in US Children Despite Pneumococcal Conjugate Vaccine,” Pediatrics, Vol. 125, No. 1, 2010, pp. 2633. doi:10.1542/peds.2009-0184

[2] I. Obando, C. Muñoz-Almagro, L. A. Arroyo, D. Tarrago, D. Sanchez, D. Moreno-Perez, et al., "Pediatric Parapneumonia Empyema, Spain,” Emerging Infectious Diseases Journal, Vol. 14, No. 9, 2006, pp. 1390-1397. doi:10.3201/eid1409.071094

[3] M. Hortal, G. Sehabiague, T. Camou, I. Iraola, M. Estevan and M. Pujadas, "Pneumococcal Pneumonia in Hospitalized Uruguayan Children and Potencial Prevention with Different Vaccine Formulations,” Journal of Pediatrics, Vol. 152, No. 6, 2008, pp. 850-853. doi:10.1016/j.jpeds.2007.11.008

[4] M. A. Fletcher, "More Severe Pneumococcal Pneumonia in Hospitalized Uruguayan Children,” Journal of Pediatrics, Vol. 154, No. 2, 2009, pp. 307-3028.

doi:10.1016/j.jpeds.2008.09.020

[5] M. Hortal, M. Estevan, I. Iraola and B. De Mucio, “A Population-Based Assessment of the Disease Burden of Consolidated Pneumonia in Hospitalized Children under five Years of Age," International Journal of Infectious Diseases, Vol. 11, No. 3, 2007, pp. 273-277. doi:10.1016/j.ijid.2006.05.006

[6] World Health Organization, Pneumonia Vaccine Trial Investigators Group, "Standardization of Interpretation of Chest Radiographs for the Diagnosis of Pneumonia in Children,” WHO, Geneva, 2001.

[7] C. L. Byington, K. Korgenski, J. Daly, K. Ampofo, A. Pavia and E. O. Mason, "Impact of the Pneumococcal Conjugate Vaccine on Pneumococcal Parapneumonia Empyema," The Pediatric Infectious Disease Journal, Vol. 25, No. 3, 2006, pp. 250-254. doi:10.1097/01.inf.0000202137.37642.ab

[8] R. T. Chen and W. A. Orenstein, "Epidemiologic Methods Inimmunization Programs,” Epidemiologic Reviews, Vol. 18, No. 2, 1996, pp. 99-113.

doi:10.1093/oxfordjournals.epirev.a017931

[9] Centers for Diseases Control and Prevention, "Pneumonia Hospitalizations among Young Children before and after Introduction of Pneumococcal Conjugate Vaccine. United States 1996-2006," Morbidity and Mortality Weekly Report, Vol. 58, 2009, pp. 1-4.

[10] K. D. Schultz, L. L. Fan, J. Pinsky, L. Ochoa, E. O’Brian Smith, S. L. Kaplan, et al., "The Changing Face of Pleural Empyema in Children: Epidemiology and Management," Pediatrics, Vol. 113, No. 6, 2004, pp. 1735-1740. doi:10.1542/peds.113.6.1735 